\title{
Opportunities and challenges when pooling milk samples using ELISA
}

\author{
Græsbøll, Kaare; Andresen, Lars Ole; Hisham Beshara Halasa, Tariq; Toft, Nils
}

Published in:

Preventive Veterinary Medicine

Link to article, DOI:

10.1016/j.prevetmed.2016.08.001

Publication date:

2017

Document Version

Peer reviewed version

Link back to DTU Orbit

Citation (APA):

Græsbøll, K., Andresen, L. O., Hisham Beshara Halasa, T., \& Toft, N. (2017). Opportunities and challenges when pooling milk samples using ELISA. Preventive Veterinary Medicine, 139(Part B), 93-98.

https://doi.org/10.1016/j.prevetmed.2016.08.001

\section{General rights}

Copyright and moral rights for the publications made accessible in the public portal are retained by the authors and/or other copyright owners and it is a condition of accessing publications that users recognise and abide by the legal requirements associated with these rights.

- Users may download and print one copy of any publication from the public portal for the purpose of private study or research.

- You may not further distribute the material or use it for any profit-making activity or commercial gain

- You may freely distribute the URL identifying the publication in the public portal

If you believe that this document breaches copyright please contact us providing details, and we will remove access to the work immediately and investigate your claim. 


\section{ABSTRACT}

(1)

\section{Opportunities and challenges when pooling milk samples using ELISA}

National Veterinary Institute, Technical University of Denmark, Bülowsvej 27, 1870 Frederiksberg C.

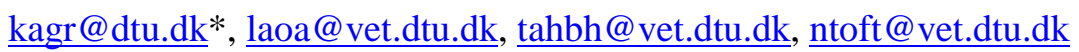

*corresponding author.

8 Testing large quantities of samples in order to detect one or more test-positive sample(s) is expensive and time-consuming. It is possible to optimize this process by pooling samples. Two frameworks to produce different hierarchical and non-hierarchical pooling schemes were tested and compared to standard pooling. Their efficiency and the potential savings were determined as a function of prevalence and the number of pooled samples.

The potential benefit of pooling samples is dependent upon the changes in the analytical sensitivity and specificity of the test used when diluting test-positive samples by pooling. To illustrate this, the sensitivity of antibody ELISA on pooled samples of bovine milk for Salmonella Dublin, Mycobacterium avium spp. paratuberculosis, and bovine virus diarrhea was tested. For these milk assays, the analytical sensitivity decreased rapidly with increasing pool sizes.

The efficiency of pooling is usually only measured by the number of tests performed, yet real savings depend on all the costs involved in the pooling process. These may differ between laboratories depending on the available equipment and the salaries of the technicians, among other factors. Therefore, several cost parameters were introduced to describe the total cost and thereby calculate the total savings. In terms of overall savings, both tested schemes were potentially optimal depending on the prevalence, possible pool size, and the cost of retesting. For the pool sizes of interest in this study, 
the three-stage hierarchical pooling scheme was often marginally more efficient in terms of the total number of tests. However, if the price of re-pooling was high, the two-stage scheme performed better in terms of total savings. In addition, for low prevalences and the possibility of pooling a large number of samples, the two-stage non-hierarchical test may be more efficient, both in terms of number of tests and overall cost. In order to apply these results in different laboratory settings, a free Shiny WebApp was developed, to compare several pooling schemes with different cost parameters.

\section{KEYWORDS}

Pooling; group testing; hierarchical; shifted transversal design

\section{ABBREVIATIONS}

DD: Double Dorfman; STD: Shifted Transversal Design

\section{INTRODUCTION}

The pooling of sample material is used extensively within the veterinary field to determine farm status and/or as an indicator for further investigation. The bulk tank milk of dairy cows can be tested for pathogens such as Salmonella Dublin (Nielsen et al., 2005), Streptococcus agalactiae (Andersen et al., 2003), bovine viral diarrhoea virus (Bitsch et al., 2000), and bovine herpesvirus (Nylin et al., 2000). However, pooling has rarely been used to detect disease in individual animals.

The minimum number of pooled tests required to detect a single test-positive sample firstly depends upon the degree to which a test-positive sample can be diluted and still be detected: how the sensitivity changes with the pool size. Secondly, it depends upon the number of test-negative samples amongst which the test-positive sample hides: the prevalence. 
47 When determining whether pools are test positive or negative, it can be beneficial to establish 48 alternative cut-offs for the ELISA, that are lower than the defaults recommended by the manufacturer.

49 A lower cut-off is required because pooling dilutes test-positive samples, causing a lower signal, which then decreases the sensitivity of the test. Lowering the cut-off is also a method of increasing the potential pool size.

Hierarchical pooling is done in multiple stages, but each sample is pooled only once per stage. For example, traditional pooling occurs in two stages, where samples are first pooled, and then samples from test-positive pools are tested individually. In a three-stage method, samples are initially pooled and samples from test-positive pools are then divided into a number of smaller pools. It is only following this second pooling stage that individual samples from positive second-stage pools are tested. This type of three-stage pooling is currently being used in HIV screening in several laboratories in the USA (Sherlock, 2007).

Pooling of samples can also be done in non-hierarchical structures of different dimensionality. Nonhierarchical means that all pools are tested simultaneously; ideally, it would be unnecessary to retest in order to locate test-positive samples. Examples of non-hierarchical pooling schemes are: pooling in two dimensions (which involves arranging samples in a matrix and pooling on the edges, so that each sample occurs in a row and column pool simultaneously), and arranging samples three dimensionally in a cube and then pooling on the edges where each sample occurs in three pools. Theoretically, this principle can be extended into infinitely high dimensions. However, there is a risk that the number of test-positive samples will exceed the non-hierarchical capacity of the test, and suspected test-positive samples in test-positive pools will then need to be retested individually to confirm the suspicion. An additional option of non-hierarchical schemes is to add extra pools, or 'layers' - this is known as combinatorial testing or solving the 'group-testing problem'. The advantage of using combinatorial pooling is that the probability of needing to retest samples to identify the individual test-positive sample may be significantly reduced. Reducing the number of retests can potentially save time and money. 
The efficiency of pooling in terms of number of tests has been the subject of a number of publications, for example many of these pooling schemes are presented in Du \& Hwang (2006), Hughes-Oliver (2006), and Cheng \& Du (2008). The relevance of a particular pooling framework may not be solely determined by the number of tests required to detect a test-positive sample, but should also include the cost of pooling, testing, re-pooling, retesting, as well as the time taken for each of these steps. Therefore, the objective of this paper is to compare pooling frameworks in terms of all the costs in addition to the number of tests performed, in order to determine the possible opportunities in terms of savings and profits, and the challenges that must be overcome to achieve those benefits.

\section{MATERIALS AND METHODS}

\section{$\underline{\text { ELISA }}$}

The sensitivity of commercially available ELISA on pooled bovine milk samples for the detection of antibodies for Salmonella Dublin (SD) (PrioCHECK Salmonella AB bovine Dublin, Prionics, Switzerland), Mycobacterium avium spp. paratuberculosis (PTB) (ID Screen Paratuberculosis Indirect, IDVet, Grabels, France), and bovine virus diarrhea (BVD) virus (Svanovir BVDV-Ab, Svanova, Uppsala, Sweden) was evaluated using the following approach. The commercially available ELISA tests were performed according to the manufacturer's instructions. Milk samples that were previously analyzed by ELISA and shown to be test positive or test negative were kindly supplied by Eurofins Steins Laboratory, Vejen, Demark. The milk samples were taken from Danish farms as part of the surveillance programs for SD, PTB, and BVD. The numbers of test-positive milk samples included in the study were: 9 for SD, 8 for PTB and 10 for BVD. Test-positive milk samples were pooled with known test-negative milk samples, resulting in one test-positive sample being pooled with 4, 9, 24, 49, 99, 149 and 199 test-negative samples. An equal volume from each sample was used for pooling. The optical density (OD) was measured at $450 \mathrm{~nm}$. Results were calculated as percent positivity (PP) using Eq. (1). 


$$
\mathrm{PP}=100 \cdot\left(\mathrm{OD}_{\text {sample }}-\mathrm{OD}_{\text {negative control }}\right) /\left(\mathrm{OD}_{\text {positive control }}-\mathrm{OD}_{\text {negative control }}\right)
$$

99

The specificity of the ELISA and alternative cut-offs for milk samples were estimated by testing 460 known test-negative milk samples in each of the three ELISA. The alternative cut-off was calculated as the mean percent positivity relative to the positive control of the assay, plus three times the standard deviation.

\section{POOLING SCHEMES}

When presenting and deriving the pooling schemes, perfect sensitivity and specificity of the test are assumed. The effect of pooling on sensitivity and specificity is elaborated in the discussion section.

1D: This is the traditional pooling scheme, and it has been used since 1915 (Hughes-Oliver, 2006). Each sample is pooled once with a number of other samples. If a pool is test positive, all samples belonging to this pool must be retested to identify the test-positive sample(s). In the 1D pooling scheme, retesting is always carried out when there are test-positive pools. This means that a 1D pooling scheme can be expensive if retesting carries a large cost. Dorfman (1943) derived an analytical expression for the expected mean number of tests, $E(T)$, given the prevalence, $p$.

$$
E_{1 D}(T)=\frac{N}{n}+p^{\prime} \frac{N}{n} n
$$

Where $N$ samples are mixed in pools of size $n$, the $N / n$ pools are tested, and the samples from the $p^{\prime} N / n$ test-positive pools are tested individually (where $p^{\prime}=1-(1-p)^{n}$ is the probability of a testpositive pool). This expression can be used to find the optimal pool size and hence the minimum number of tests required for a given prevalence. However, this paper includes all combinations of pool sizes and prevalences, as the optimum pool size may change according to the different costs associated with the pooling and testing process.

STD: The Shifted Transversal Design was introduced by Thierry-Mieg (2006). This framework was originally intended as a non-hierarchical scheme, meaning that test-positive samples could be found using only one stage without retesting. However, ensuring one-stage functionality requires additional 
121

122

123

124

pools. In this paper, the STD is tested in both a one- and two-stage mode, because the optimal mode depends on the cost of retesting. The STD includes traditional 1D testing, methods similar to the twoand three-dimensional methods mentioned in the introduction, as well as higher dimensions. Furthermore, it is a combinatorial pooling scheme, i.e. additional pools are created with a minimal cooccurrence of samples in each pool. Minimizing co-occurrence means that the STD can detect multiple test-positive samples with a reduced probability of retesting. In the STD, the number of different pooling combinations with a similar pool size increases with pool size. For a pool size of 36, there are 166 different pooling schemes of different dimensions and combinatorial complexity. The optimal pooling scheme can be determined from these by applying the costs associated with the pooling scheme steps, and comparing these costs to the individual testing. For the complete mathematical description of the STD, see Thierry-Mieg (2006). In the original paper, the STD was used in a strictly non-hierarchical (one-stage) mode by imposing the theoretical minimum number of layers given by Corollary 3 in Thierry-Mieg (2006). In this paper, the restriction is lifted and we test the STD for all number of layers from one to the maximum possible, as defined by the model.

When working in one-stage mode, no retest is necessary for the STD, and the efficiency is easily calculated. However, an increasing number of test-positive samples necessitate the use of the twostage mode, and the multiple possible combinations of test-positive sample locations within the pooling structures give rise to a very complicated probabilistic structure determining the number of retests required. Therefore, the pooling schemes were simulated to determine the average number of retests. The simulation method was as follows: all possible pooling schemes with a pool size $\leq 36$ were tested in combination with prevalences from $0.1 \%$ to $90 \%$. For each combination of pool size and prevalence, the number of individual positive samples was drawn from a binomial distribution. This was repeated 100 times for the STD. The number of times to retest and the average number to be retested were saved for each combination of pool size and prevalence.

DD: This is a three-stage hierarchical pooling scheme with variable pool size. This framework will be referred to here as the 'Double Dorfman' (DD), as the analytical expression is based on derivations by 
147

Dorfman (1943). In the first stage, $N$ samples are mixed in pools of size $n_{l}$, the $N / n_{l}$ pools are tested, and the samples from the $p^{\prime} N / n_{1}$ test-positive pools are mixed again in pools of size $n_{2}$, where $p^{\prime}=1-(1-p)^{n 1}$ is the probability of a test-positive pool, and $p$ is the prevalence of test positives in the $N$ samples. Lastly, samples from the test-positive pools in the second stage are individually tested. The expected mean number of tests, $E_{D D}(T)$, is expressed as:

$$
E_{D D}(T)=\frac{N}{n_{1}}+p^{\prime} \frac{N}{n_{1}}\left(\frac{n_{1}}{n_{2}}+n_{2} \frac{n_{1}}{n_{2}}\left(1-\left(1-\frac{p}{p^{\prime}}\right)^{n_{2}}\right)\right)
$$

Reducing this expression and dividing by $N$ gives the fraction of tests required, $C$ :

$$
C=\frac{1}{n_{1}}+p^{\prime}\left(\frac{1}{n_{2}}+\left(1-\left(1-\frac{p}{p^{\prime}}\right)^{n_{2}}\right)\right)
$$

This expression only takes into account the number of tests. However, there may be further costs associated with the additional stage of testing. Therefore, the number of pools, re-pools, and retests were calculated for every combination of first and second pool size from 2 to 36 , in combination with prevalences from $0.1 \%$ to $90 \%$. For an initial pool size of 36 , there were 35 different stage-two pool sizes. The optimal pooling scheme can be selected from these by applying the costs and comparing them to the individual testing strategy.

Comparison of pooling schemes: When comparing pooling schemes to individual sample testing, the simple comparison is to count the total number of tests required to detect the test-positive samples. However, there are costs associated with the pooling itself and/or the storage and preparation involved in retesting the individual samples identified as possible test positives by the pooling schemes. In order to account for the price of complex pooling schemes, the costs examined were: the cost of testing a single sample (which was set to index one); the price of retrieving a batch of samples that were in a test-positive pool (the retrieval cost); an alternative measure of the re-pooling cost, where the cost is per retested sample, and the hourly cost of the robot used to pool the samples. To complete the total cost, it was also necessary to specify the time taken to pool and test the samples. When this is 
included, it is also possible to calculate the profit gains per time unit, since a scheme that reduces the number of tests may be slower or faster in time, affecting the overall profitability. Examples of savings and profit calculations have been included in the supplementary material.

For simplicity, it was assumed that the test used in the simulations had $100 \%$ sensitivity and specificity ( $\mathrm{Se} / \mathrm{Sp})$. The impact of non-perfect tests is elaborated in the discussion.

All calculations and simulations were performed using R: A Language and Environment for Statistical Computing ver. 3.1.1 (R development Core Team, 2014) in RStudio ver. 0.99.447 (RStudio Team, 2015).

\section{RESULTS}

Table 1 summarizes the results of testing negative milk samples in the three ELISA. Results of pooling the nine SD test-positive milk samples with test-negative milk samples are presented in Fig. 1. Similar results were obtained when testing milk samples using assays for antibodies to PTB and BVD virus. Results show a large decrease in the percent positivity (PP) when test-positive samples are pooled with an increased number of test-negative samples. Pool sizes of more than 25 samples containing one test-positive sample showed results at the level of the test-negative samples. A maximum pool size of five would give test-positive results with the alternative cut-off of $21 \mathrm{PP}$, as shown in Fig. 1. Pool sizes greater than five could result in false test-negative measurements for milk. 
Table 1. Results from a test of the test-negative milk samples in ELISA.

\begin{tabular}{lllllll}
\hline $\begin{array}{l}\text { ELISA } \\
\text { for antibodies to * }\end{array}$ & $\begin{array}{l}\text { Negative } \\
\text { samples } \\
\text { tested }\end{array}$ & $\begin{array}{l}\text { Mean } \\
\text { Percent } \\
\text { Positivity }\end{array}$ & $\begin{array}{l}\text { Standard } \\
\text { deviation }\end{array}$ & $\begin{array}{l}\text { Manufacturer's } \\
\text { cut-off }\end{array}$ & $\begin{array}{l}\text { Alternative } \\
\text { cut-off }\end{array}$ & $\begin{array}{l}\text { Specificity } \\
\text { using } \\
\text { alternative } \\
\text { cut-off }\end{array}$ \\
\hline Salmonella Dublin & 460 & 6.65 & 4.62 & 35 & 21 & 0.99 \\
$\begin{array}{l}\text { Mycobacterium } \\
\text { avium spp. } \\
\text { paratuberculosis }\end{array}$ & 460 & 2.00 & 2.18 & 15 & 9 & 0.995 \\
$\begin{array}{l}\text { Bovine Diarrhea } \\
\text { Virus }\end{array}$ & 460 & 3.50 & 0.93 & 12 & 7 & 0.99 \\
\hline *ELISA used are presented in materials and methods. & & & & & \\
\hline
\end{tabular}

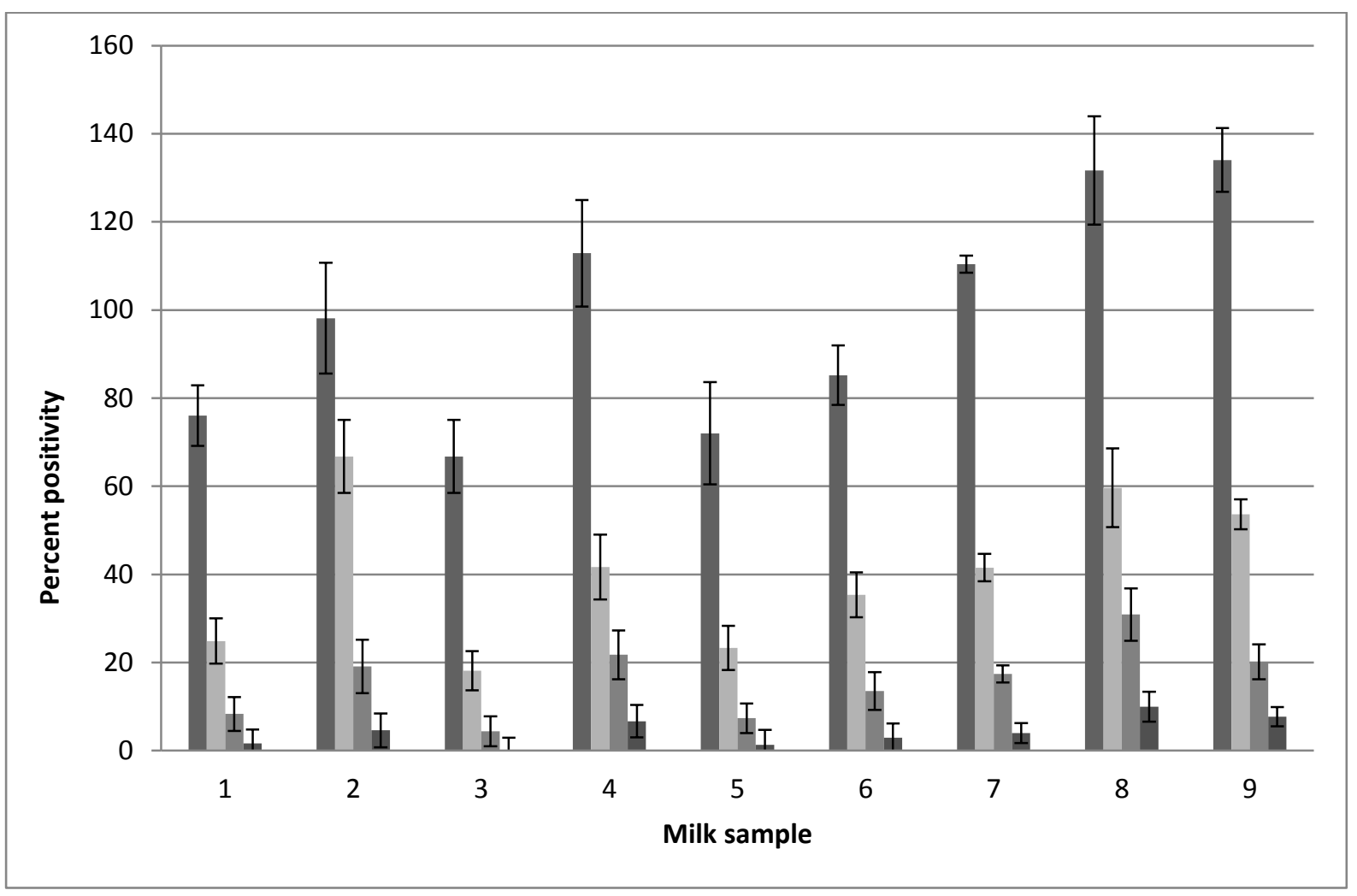

Fig. 1: Salmonella Dublin ELISA: Percent positivity in pooled milk samples. Nine antibody testpositive samples and one antibody test-negative sample were pooled with known test-negative milk samples. Results from testing the milk samples undiluted (dark gray), diluted 1:5 (light gray), 1:10

194 (gray) and 1:25 (black) are presented as mean values of three tests performed on separate days. Standard errors are indicated. 
196

197 The results of the simulations show that the DD is more efficient when considering only the number

198 of tests required (Figure 2). However, when introducing a retrieval cost, the STD becomes more cost-

199 efficient than DD (Figure 3). Both the STD and DD show potentially large savings for pool sizes 200 greater than four, providing the prevalence is low enough.

201

202 
1D
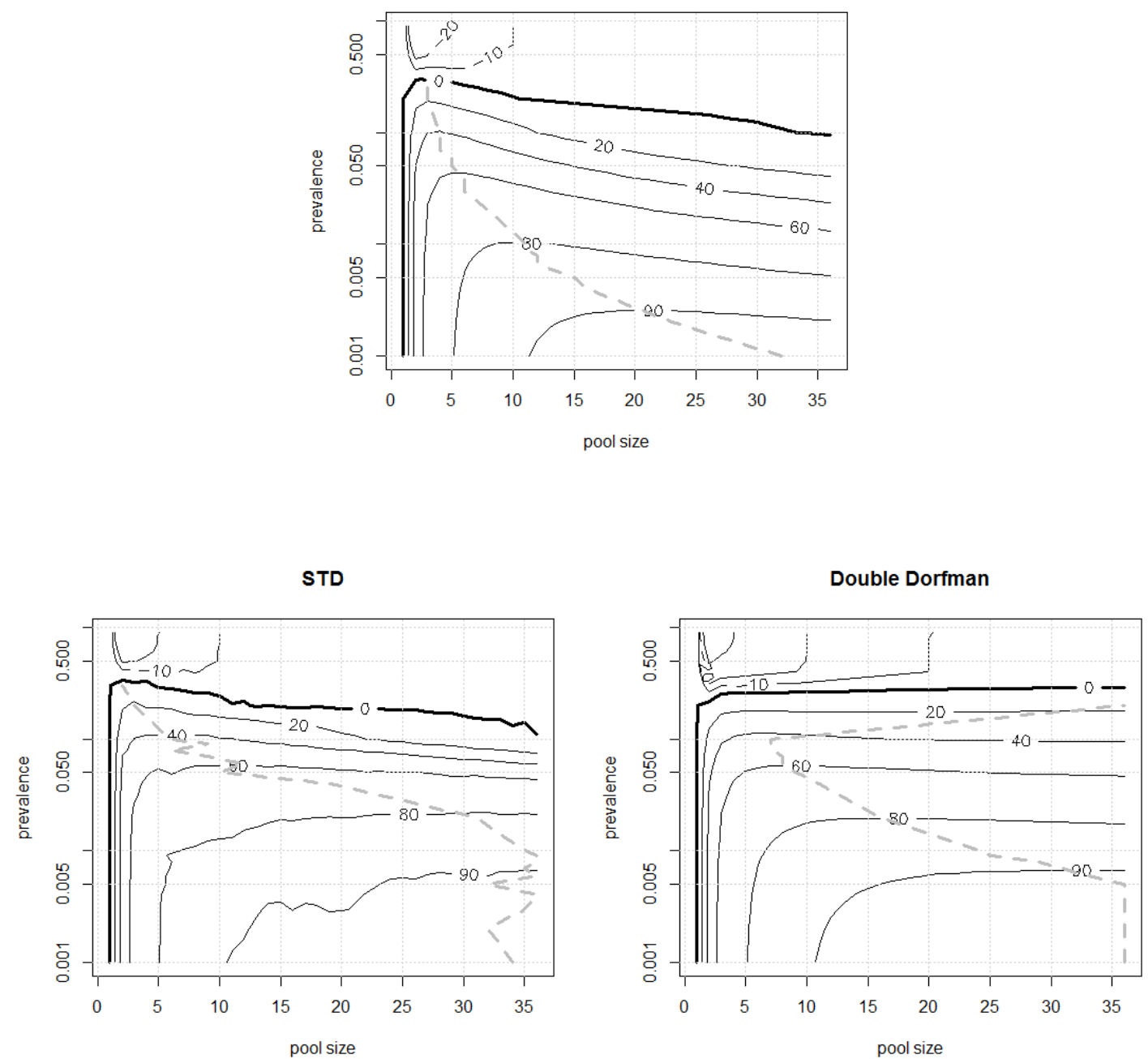

Fig. 2: Contour plots of the fraction of tests saved compared to individual testing as a function of pool size and prevalence. The thick line indicates where the pooling scheme requires the same number of tests as individual testing, and above this line pooling is not cost effective. The dashed gray line indicates the optimal pool size for a given prevalence, (i.e., the pool size that gives the maximum percentage of saved tests). This plot is equivalent to a savings plot where all costs (except the cost of testing) are set to zero. 

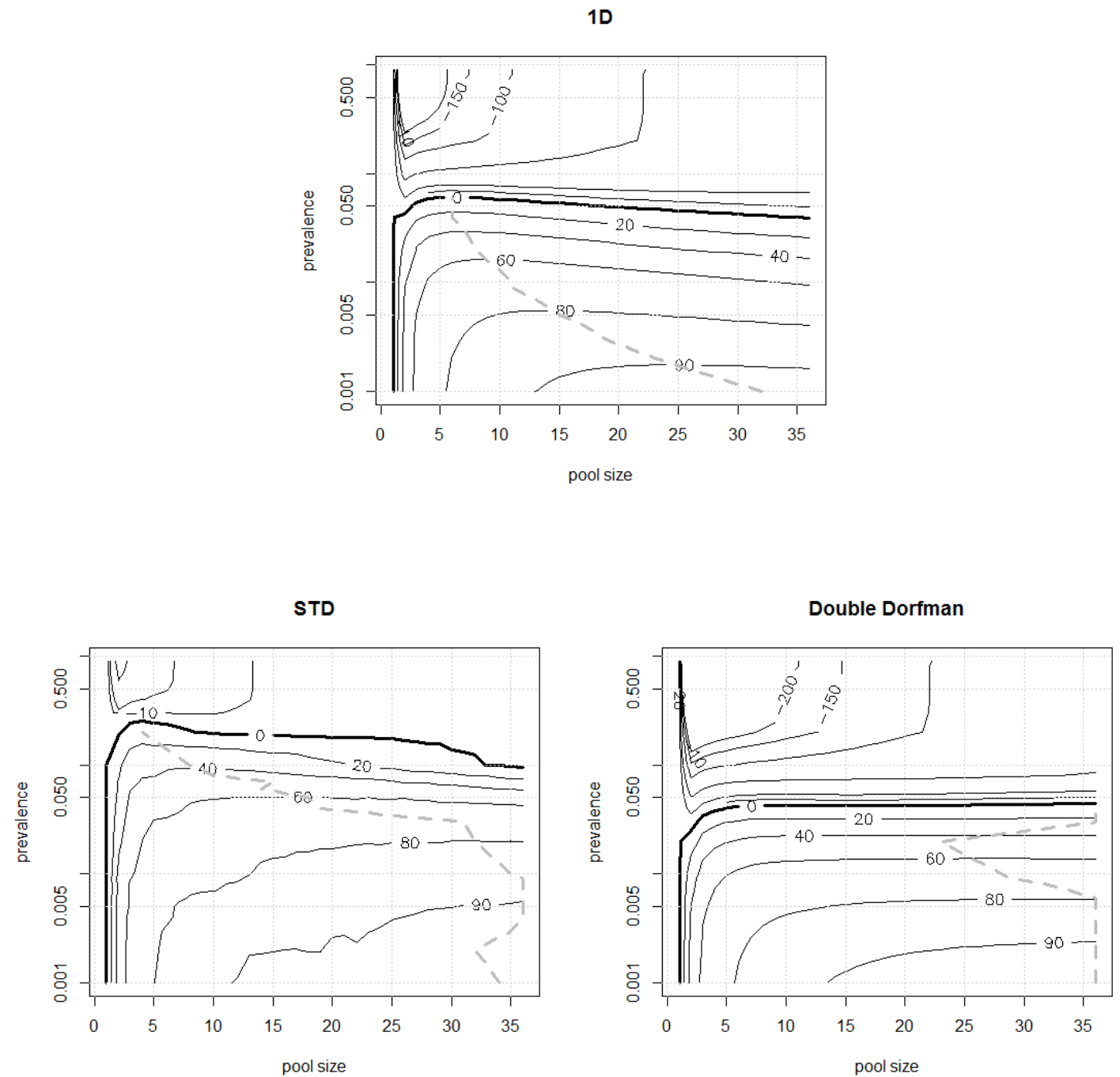

213 Fig. 3: Contour plots of the fraction of savings compared to the price of individual testing as a

214 function of pool size and prevalence, where each batch retrieval for retesting caries the cost of 10

215 individual tests. The thick line indicates where the pooling scheme has the same cost as individual

216 testing, and above this line pooling is not cost effective. The dashed gray line indicates the optimal

217 pool size for a given prevalence (i.e., the pool size that gives the maximum percentage of saved costs).

218 For all pooling schemes, there is an optimal pool size for a given prevalence (dashed gray lines in

219 Figures 2 and 3). It is also apparent that pooling is not efficient when the prevalence is higher than $30 \%$ (Figures 2 and 3). 
221 For the small pool sizes used in this study, the DD performs best in terms of the number of tests

222

223

224

225

226

227 required. The parameter space explored here covers both high prevalence and limited pool size, which includes complicated situations with multiple test-positive samples in each pool. At the other end of the parameter set, an approximate solution can be derived for the situation where the STD is better (in terms of fewer samples tested) than DD, when the pool size, $n$, is larger than $((d-1) /(2 p))^{2 / 3}$ (where $p$ is the prevalence, and $d$ is the dimension of the pooling scheme). This is valid when $p n^{d /(d-1)}<<1$ and $n \geq 2^{d /(d-1)}$, for any integer value of $d \geq 2$. Fewer samples are tested and the retrieval cost in the STD is lower, so therefore the total cost will also be lower, regardless of the specific cost parameters chosen. The mathematical derivation is available in the supplementary material.

\section{DISCUSSION}

The profitability of implementing pooling in a laboratory is dependent upon many laboratory-specific costs. In this paper, the relative cost of testing and retesting was shown to be the most important factor when determining the most profitable pooling scheme. The DD required fewer tests than the STD within the tested parameter space. However, if the costs associated with retrieval at batch level are even marginally larger than the cost of a single test, the STD scheme results in larger savings. In addition, the STD can be shown to be superior to the DD for low prevalence and large pool sizes. Both the DD and the STD are always superior to or equally efficient as the traditional 1D pooling in terms of number of tests.

The specific costs of testing or re-pooling/retesting may differ between laboratories. In this paper, a rather general cost structure was assumed, where the cost of testing includes the cost of a single test, the cost of a robot/technician to perform the pooling, and the time taken for both of these processes. The cost of retesting includes the cost of retrieving samples from a test-positive pool to be repooled/retested. The time taken to retrieve samples was not included, as it was assumed that the number of samples was large enough that samples for re-pooling/retesting could be retrieved 

simultaneously with the testing of other samples. Therefore, the retrieval of samples is never a bottleneck, while the pooling and testing of samples was dependent upon the capacity of the facility.

248 Although changing the parameters within the testing or retesting group changed the overall savings slightly (not shown), the only large effect was when changing the two groups relative to each other, as seen in Figures 2 and 3. It was assumed that testing samples took longer than pooling. Given that different laboratories may have different cost structures, all results from simulations were collected in a WebApp that allows for the input of cost parameters. This WebApp is available online (https://dtuvetepi.shinyapps.io/SMARTPOOL/) and the source code is available as supplementary material.

The best practice for implementing these pooling schemes may be highly dependent upon the equipment available in a specific laboratory. The DD scheme can be achieved by conventional pipetting (e.g. 'by hand'), but both the DD and the STD are more easily performed by a robot. The robot should not arrange samples in the physical structures, but rather receive a list of pools to which each sample is assigned. In this way, each individual sample is only visited once, and is directly distributed to the relevant number of pools determined by the pooling scheme. However, the best practice and the cheapest pooling method may depend on the specific laboratory.

From the ELISA dilution trials, it was evident that pooling impacts the $\mathrm{Se} / \mathrm{Sp}$, which were not specifically addressed in the simulations. Instead, the results of the simulations in this paper were reported as a function of the pool size, which may allow a user to choose a pool size with a desired Se/Sp based on their own dilution trials using a desired ELISA. To use the results in such a way, it must be assumed that $\mathrm{Se} / \mathrm{Sp}$ is sample-specific (i.e. there is perfect repeatability): a sample that tests negative in a pooled test will test negative in all pooled tests of the same pool size, and the same for positive tests. The Se/Sp for a given disease using a specific test kit can be determined by dilution trials, as presented in this paper. Here, pooling of negatives was also used to determine an alternative cut-off in order to maximize the possible pool size. When choosing a pool size for a multi-stage pooling scheme, it can be beneficial to choose an alternative lower cut-off in the ELISA test to 
increase sensitivity and/or pool size. The apparent loss of specificity seen when lowering the cut-off can be negated by performing the retest of individual samples using the manufacturer's cut-off. This is because false test positives will then be retested subject to the manufacturer's kit specificity. As a further means of increasing sensitivity, it may also be possible to adjust the procedure of the ELISA if there are pre-dilution steps before the OD measurements, as suggested by Brinkhof et al. (2007).

The dilution experiments presented in this paper are examples - a larger sample size of test-positive samples is required to give a better prediction of the change in sensitivity when pooling. Specifically, weak test-positive samples must be included in the test series in order to accurately estimate the changes in sensitivity due to pooling. Specifically, tests should be carried out to ensure that the randomness of the test does not increase around the cut-off, as reported i.e. for PCR tests with low integrity (Fleige \& Pfaffl, 2006). Furthermore, it should be assessed whether samples could give rise to added unspecific reactivity when pooled.

Hierarchical testing was also investigated using a halving method (results not shown). This method pools samples in an initial pool, and if this initial pool tests positive, then the samples are re-pooled into two pools and tested. For each test-positive pool, the samples are divided into two new pools and tested. This is done repeatedly to the individual sample level. This method showed similar performance to the DD in terms of the number of tests, but since there are more stages (providing the initial pool size is greater than four), then this method was not superior to the DD in terms of costs.

Black et al. (2015) presented an optimized framework to test samples with heterogeneous prevalences at the individual level. This could be utilized within the veterinary field if risk factors were known about the individual cows. An example could be the age-dependent sensitivity of testing for Mycobacterium avium ssp. paratuberculosis (Nielsen et al., 2013). This does, however, require the extensive sorting of the samples, or the possibility of simultaneous pooling to several pools. Another use of prior knowledge may be at herd level, so that the expected prevalence in the herd (which could be determined from a previous round of testing) would determine the pooling scheme, given the results from this paper. If there is no prior knowledge of the prevalence, it could also be possible to 
298

use the first-stage testing to estimate the prevalence at herd level, and use this estimate to determine the pool size of the second stage.

This paper expands on work presented at the annual meeting of SVEPM 2016 (Græsbøll et al., 2016), explicitly comparing 1D and higher dimensional pooling to the STD, which showed that the STD was always superior. Future work on this subject could investigate a combination of the two types of testing, including testing where prevalence is estimated after stage two to determine the pool size in stage three. It is also possible that dividing into test positive and test negative removes information from the test, and it may be advantageous and/or easier to make algorithms to identify test-positive samples based on the distribution of the continuous outcomes in a pooling scenario.

The code used in this paper has been integrated into a Shiny WebApp, which allows the user to specify the cost parameters and different prevalence ranges to investigate the impact on savings and profit across parameter space to emulate different laboratory settings. The WebApp is freely available at: https://dtuvetepi.shinyapps.io/SMARTPOOL/. The R code for the Shiny WebApp has also been included as supplementary material, so that it may be run on a local machine.

\section{$\underline{\text { CONCLUSION }}$}

Large savings can be achieved with pooling. There is a potential to reduce the test requirements by up to $80 \%$, even with pool sizes as small as 5 , if the prevalence is low. However, there are certain restrictions that apply before those savings can be accomplished. Firstly, the expected prevalence of individual samples must be lower than $30 \%$, otherwise pooling is not efficient. Secondly, initial tests must be performed on the specific test kit intended for use, and ideally in combination with a defined alternative cut-off to maximize $\mathrm{Se} / \mathrm{Sp}$, in order to determine how large a pool size can be used. Thirdly, samples should not display an increase in any unspecific reactivity in the test when pooled, otherwise results may be invalid. The optimal pooling framework depends on the costs associated 
322

323

324

325

326

327

328

329

330

331

332

333

334

335

336

337

338

339

340

341

342

343

with pooling; in particular, the cost of identifying samples for retesting or re-pooling influences the optimal pooling scheme.

\section{ACKNOWLEDGEMENTS}

All milk samples were kindly supplied by Eurofins Steins Laboratorium, Vejen, Denmark. This work was carried out as a part of the SMARTPOOL project which is funded by GUDP, grant no. 34009-13-0760. The funder had no influence on the design, execution, results, or conclusions of the experiments or simulations in this study.

\section{DECLARATIONS}

Some of the work referred to in this manuscript has previously been presented by the authors at the annual meeting of SVEPM 2016, which includes a conference paper.

\section{REFERENCES}

Andersen, H. J., Pedersen, L. H., Aarestrup, F. M., \& Chriél, M. (2003). Evaluation of the surveillance program of Streptococcus agalactiae in Danish dairy herds. Journal of dairy science, 86(4), 1233-1239.

Barillot, E., Lacroix, B., \& Cohen, D. (1991). Theoretical analysis of library screening using a Ndimensional pooling strategy. Nucleic acids research, 19(22), 6241-6247

Bitsch, V., Hansen, K. E., \& Rønsholt, L. (2000). Experiences from the Danish programme for eradication of bovine virus diarrhoea (BVD) 1994-1998 with special reference to legislation and causes of infection. Veterinary microbiology, 77(1), 137-143. 
Black, M.S., Bilder, C.R., \& Tebbs, J.M. (2015). Optimal retesting configurations for hierarchical group testing. Journal of the Royal Statistical Society: Series C (Applied Statistics)

346 Brinkhof, J.M.A., Houwers, D.J., \& Van Maanen, C. (2007). Development of a sample pooling 347 strategy for the serodiagnosis of small ruminant lentiviral infections using the ELITEST-MVV ELISA. Small Ruminant Research, 70(2), 194-199

Cheng, Y., \& Du, D. Z. (2008). New constructions of one-and two-stage pooling designs. Journal of Computational Biology, 15(2), 195-205.

Dorfman, R. (1943) The detection of defective members of large populations. Ann. Math. Statist., 14, $436-440$.

Du, D., \& Hwang, F. (2006). Pooling designs and nonadaptive group testing: important tools for DNA sequencing (Vol. 18). World Scientific Publishing Company Incorporated.

Fleige, S., \& Pfaffl, M. W. (2006). RNA integrity and the effect on the real-time qRT-PCR performance. Molecular aspects of medicine, 27(2), 126-139.

Græsbøll, K, Andresen, L.O., Halasa, T., Toft, N. How few pooled tests are needed to detect a single positive sample? SVEPM 2016 proceedings.

Hughes-Oliver, J. (2006). Pooling experiments for blood screening and drug discovery. Screening: paratuberculosis antibody response through age. PLoS ONE, 8, e63009 
365 Nylin, B., Strøger, U. and Rønsholt, L. (2000). A retrospective evaluation of a Bovine Herpesvirus-1 366 (BHV-1) antibody ELISA on bulk-tank milk samples for classification of the BHV-1 status of Danish 367 dairy herds. Prev. Vet. Med. 47, 91-105

368 R Development Core Team. (2014). R: A Language and Environment for Statistical Computing. R

369 Foundation for Statistical Computing. Vienna, Austria. www.R-project.org

370 RStudio Team. (2015). RStudio: Integrated Development Environment for R. RStudio, Inc. Boston,

371 MA. www.rstudio.com

372 Sherlock, M., Zetola,N. and Klausner, J. (2007) Routine detection of acute HIV infection through

373 RNA pooling: Survey of current practice in the United States. Sexly Transmttd Dis., 34, 314-316.

374 Thierry-Mieg, N. (2006). A new pooling strategy for high-throughput screening: the Shifted 375 Transversal Design. BMC bioinformatics, 7(1), 28 